\title{
Time for biocide stewardship?
}

Authors: Roxana Zamudio ${ }^{a}$, Marco R Oggionia, Ian M Gould ${ }^{b}$ and Karolin Hijazi ${ }^{*}$

Addresses: aDepartment of Genetics and Genome Biology, University of Leicester, Leicester, UK; b Department of Medical Microbiology, Aberdeen Royal Infirmary, Aberdeen, UK; ' School of Medicine, Medical Sciences \& Nutrition, University of Aberdeen, Aberdeen, UK,

${ }^{*}$ Corresponding author. Tel: +44 (0) 1224-555153, e-mail address k.hijazi@abdn.ac.uk

Word count: 979 words (includes reference numbering)

Figures and tables: 0 
To the Editor - we wish to comment on the article by Lee et al ${ }^{1}$. We welcome this important work emphasising the spread of pathogenic multidrug resistance Staphylococcus epidermidis globally but wish to highlight some critical findings derived from the deposited genomic data which have been partially reported in the supplementary information (Supplementary Table 1, sheet D) but not discussed in the article.

The study by Lee et al supports the concerns raised in numerous smaller scale and local studies that $S$. epidermidis is a dominant reservoir of multidrug resistance genes ${ }^{2}$. The authors' main focus is the prevalence of rpoB associated with resistance to rifampicin widely used in medical devices. We wish to draw the readers' attention to the high carriage of biocide resistance genes as well as heavy metal resistance in association with staphylococcal cassette chromosome mec (SCCmec) elements in hospital-adapted multidrug resistant $S$. epidermidis.

Biocide resistance. We focussed our analyses on i) qacA/B encoding an efflux pump for a variety of lipophilic cations and strongly associated with reduced microbial susceptibility to chlorhexidine ${ }^{3}$, ii) the plasmid-borne ileS2 gene and the core-genome V588F mutation in lleS responsible for high- and low-level mupirocin resistance, respectively ${ }^{4}$. Chlorhexidine is one of the most commonly used biocides in the community as well as hospital decontamination and infection control, with applications ranging from mouthwashes to impregnated catheters and skin/mucosal surface decontamination in intensive care units. Equally mupirocin is widely employed for decontamination of nasal surfaces in intensive care. Whilst these measures have shown remarkable effectiveness in control of bloodstream infections including those related to MRSA, MSSA and coagulase-negative Staphylococci ${ }^{5}$, resistance to mupirocin and declining susceptibility to chlorhexidine has been reported worldwide ${ }^{3,6}$. Resistance to biocides has raised concerns of selection of multidrug resistant bacteria associated with hospital-acquired infections with a most alarming report suggesting that exposure to chlorhexidine is associated with resistance to last resort antibiotic colistin ${ }^{7}$.

The prevalence of $q a c A / B$ in $S$. aureus reported over the last decade is very variable although the drivers for high carriage are not clear. While some studies continue to report declining susceptibility to chlorhexidine and increasing prevalence 
of qac $A / B$ in MRSA from a range of clinical settings ${ }^{8,9}$, other studies, including our own work, report low presence of $q a c A / B$ in $S$. aureus from screening samples and bacteraemia patients ${ }^{10,11}$. However, we have shown a high prevalence $(80 \%)$ of qacA/B in $S$. epidermidis isolates from blood cultures randomly collected over 6 years in an intensive care unit in Scotland, with qac $A / B$ carriage coinciding with reduced susceptibility to chlorhexidine ${ }^{11}$. Importantly the majority of $q a c A / B$ isolates in this study belonged to multidrug resistant clone ST2, the same lineage that dominated in the collection of isolates studied by Lee et al. We have also identified genetic determinants of resistance to mupirocin (ileS2, V588F mutation in lleS) and/or triclosan (sh-fabl, F204L mutation in Fabl) in $65 \%$ of qacA/B S. epidermidis isolates ${ }^{11}$. Notably, in some cases mupirocin resistance genes were co-located with qac $A / B$ on variants of known MRSA mobile elements raising the possibility of horizontal transfer of multidrug resistance genes between $S$. epidermidis and $S$. aureus ${ }^{11}$.

We have analysed the genomic data published by Lee et al (ENA, Bioproject PRJEB12090, PRJNA470534 and PRJNA470752) for carriage of the qacA/B genes, the lleS-V588F mutation, the ileS2(mupA) gene, the Fabl-F204L mutation and the sh-fab/gene. We did not analyse either ileS or fab/ promoter mutations, thus underestimating resistance to mupirocin and triclosan. Analysis of the 226 clinical isolates in Lee et al revealed significant enrichment of biocide resistance in the three hospital-adapted multidrug resistant lineages of this global collection of pathogenic S. epidermidis, namely ST2, ST2-BPH0662 and ST23. The proportion of qacA/B positive isolates belonging to the ST2 and ST2-BPH0662 clones (85\% and 78\%, respectively) was significantly higher ( $p=0.004$, Fisher's exact test) than that detected in all other isolates cumulatively (68\%). The ST23 and the ST2-BPH0662 lineage showed increased carriage of the core-genome lleS-V588F mupirocin resistance mutation compared to the other isolates (56\% ST23, 48\% ST2-BPH0662, $10 \%$ in other isolates; $p<0.0001$ for both lineages) but not of the plasmid-borne ileS2 (mupA) gene. There were no significant differences in the carriage rate of sh_fabl and Fabl-F204L mutations pointing to a lesser role of triclosan, a biocide primarily used in household products. 
SCCmec and heavy metal resistance. Our analysis revealed that all isolates belonging to the ST2 BPH0662 lineage described in Lee et al carry an SCCmec element orthologous to the SCCmec associated with the copper and mercury resistance mobile element (COMER) in community-associated MRSA USA300 ${ }^{12}$. In ST2 BPH0662 it presents as a composite element comprising an SCCmec type III and a COMER-like locus which, in addition to copper and mercury resistance, carries cadmium and arsenic resistance gene clusters that are not present in the MRSA SCCmec COMER element. Of note, decreased susceptibility to copper has been recently linked to increased resistance of COMER-positive $S$. aureus to macrophage killing and the success of the USA300 clone ${ }^{13}$.

The global and sustained dominance of three hospital-adapted multidrug resistant lineages with high carriage of chlorhexidine and mupirocin resistance genes raises concerns of selection of multidrug resistance by these two biocides. Further, carriage of SCCmec in association with metal resistance genes points to selection of multidrug resistant $S$. epidermidis which may display enhanced fitness and resistance to innate immunity by analogy with $S$. aureus. Indeed, we have previously demonstrated that antibiotic use is an important predictor of prevalence density of dominant multidrug resistant clones ${ }^{14}$ - we speculate a similar effect by intensive use of metals, chlorhexidine and mupirocin as previously suggested ${ }^{15}$. To support or refute this claim we recommend that future genomic analysis of hospital-acquired multidrug resistant $S$. epidermidis should include analysis of biocide resistance genes. Functional studies are required to evaluate the role of heavy metal resistance genes related to SCCmec elements in S. epidermidis.

\section{References}

1. Lee, J. Y. H. et al. Global spread of three multidrug-resistant lineages of Staphylococcus epidermidis. Nat. Microbiol. 3, 1175-1185 (2018).

2. Otto, M. Coagulase-negative staphylococci as reservoirs of genes facilitating MRSA infection: Staphylococcal commensal species such as Staphylococcus epidermidis are being recognized as important sources of genes promoting MRSA colonization and virulence. Bioessays 35, 4-11 (2013). 
3. Furi, L. et al. Evaluation of reduced susceptibility to quaternary ammonium compounds and bisbiguanides in clinical isolates and laboratory-generated mutants of Staphylococcus aureus. Antimicrob. Agents Chemother. 57, 3488-3497 (2013).

4. Hurdle, J. G., O'Neill, A. J., Mody, L., Chopra, I. \& Bradley, S. F. In vivo transfer of high-level mupirocin resistance from Staphylococcus epidermidis to methicillinresistant Staphylococcus aureus associated with failure of mupirocin prophylaxis. J. Antimicrob. Chemother. 56, 1166-1168 (2005).

5. Gould, I. M. et al. Topical antimicrobials in combination with admission screening and barrier precautions to control endemic methicillin-resistant Staphylococcus aureus in an Intensive Care Unit. Int. J. Antimicrob. Agents 29, 536-543 (2007).

6. Otter, J. A. et al. Selection for qacA carriage in CC22, but not CC30, methicillinresistant Staphylococcus aureus bloodstream infection isolates during a successful institutional infection control programme. J. Antimicrob. Chemother. 68, 992-999 (2013).

7. Wand, M. E., Bock, L. J., Bonney, L. C. \& Sutton, J. M. Mechanisms of Increased Resistance to Chlorhexidine and Cross-Resistance to Colistin following Exposure of Klebsiella pneumoniae Clinical Isolates to Chlorhexidine. Antimicrob. Agents Chemother. 61(1). pii: AAC.01162-16. doi, 10.1128/AAC.01162-16. Print 2017 Jan. (2017).

8. Cho, O. H. et al. Prevalence and Microbiological Characteristics of qacA/BPositive Methicillin-Resistant Staphylococcus aureus Isolates in a Surgical Intensive Care Unit. Microb. Drug Resist. 24, 283-289 (2018).

9. Rondeau, C. et al. Current Molecular Epidemiology of Methicillin-Resistant Staphylococcus aureus in Elderly French People: Troublesome Clones on the Horizon. Front. Microbiol. 7:31. doi, 31 (2016).

10. Hayden, M. K. et al. Chlorhexidine and Mupirocin Susceptibility of MethicillinResistant Staphylococcus aureus Isolates in the REDUCE-MRSA Trial. J. Clin. Microbiol. 54, 2735-2742 (2016).

11. Hijazi, K. et al. Susceptibility to chlorhexidine amongst multidrug-resistant clinical isolates of Staphylococcus epidermidis from bloodstream infections. Int. J.

Antimicrob. Agents (2016).

12. Planet, P. J. et al. Parallel Epidemics of Community-Associated MethicillinResistant Staphylococcus aureus USA300 Infection in North and South America. J. Infect. Dis. 212, 1874-1882 (2015).

13. Purves, J. et al. A horizontally gene transferred copper resistance locus confers hyper-resistance to antibacterial copper toxicity and enables survival of community acquired methicillin resistant Staphylococcus aureus USA300 in macrophages.

Environ. Microbiol. 20, 1576-1589 (2018). 
14. Lawes, T. et al. Turning the tide or riding the waves? Impacts of antibiotic stewardship and infection control on MRSA strain dynamics in a Scottish region over 16 years: non-linear time series analysis. BMJ Open 5, e006596-2014-006596. (2015).

15. Oggioni, M. R. et al. Significant Differences Characterise the Correlation Coefficients between Biocide and Antibiotic Susceptibility Profiles in Staphylococcus aureus. Curr. Pharm. Des. 21, 2054-2057 (2015). 